\title{
Editorial
}

\section{Journal of Parkinson's Disease - an Excellent First Year and a New Sister}

This month marks the first anniversary of the launch of the Journal of Parkinson's Disease (www.journalofparkinsonsdisease.com) and a fitting occasion for us to thank you for your support as authors, referees and readers. Thanks to the enthusiastic support of the research community, the journal has greatly surpassed our expectations. Even with such an outstanding Editorial Board behind us, we were expecting the first year (or two) to be somewhat difficult while the journal established itself.

It has been a pleasant surprise, therefore, for the journal to receive a steady stream of good quality papers, even before achieving all of the milestones a new journal must before it can really become established, such as indexing by PubMed, Scopus and WoS. We are happy to report that JPD has now been accepted for indexing in Scopus, and, having completed the requisite period of publication, is under consideration for inclusion in PubMed and WoS. Once accepted, we understand that indexing is retrospective, and we hope to have good news to announce soon in this regard.

In its first year, the journal has produced six print issues, with continuous on-line publication. We have published 35 research articles, and 10 reviews, without compromising on quality (even at this early stage the journal has a $50 \%$ rejection rate).

Given this success, it is perhaps not surprising that this anniversary month for JPD also sees the launch of a new sister, the Journal of Huntington's Disease (http://www.iospress.nl/journal/journalof-huntingtons-disease/), also dedicated to providing a forum for high quality original research in basic science, translational research and clinical medicine. The Journal of Huntington's Disease has two outstanding Editors in Chief in Blair Leavitt and Leslie
Thompson, and support from a first rate Editorial Board (seewww.iospress.com/journal/journal-ofhuntingtons-disease/?tab=editorial-board). The Journal of Huntington's Disease's first issue and all review papers published in 2012 will be freely available, and its first volume of publication (4 issues) will be included as a bonus to subscribers of the Journal of Alzheimer's Disease and/or the Journal of Parkinson's Disease.

We are delighted by this addition to the family, and given the high quality of the new arrival, we are confident that, like JPD, the Journal of Huntington's Disease will have a first year that will establish it as an influential and positive force in its field for years to come.

Best wishes

Editor-in-Chief

Patrik Brundin, MD, PhD

Van Andel Institute

Grand Rapids, MI, USA

Editor-in-Chief

J. William Langston, MD

The Parkinson's Institute and Clinical Center

Sunnyvale, CA, USA

\section{SUBMISSION OF PAPERS}

Prospective authors for Journal of Huntington's Disease can submit their manuscript electronically to the online submission system msTracker: http://mstracker.com/submit1.php?jc=jhd. 